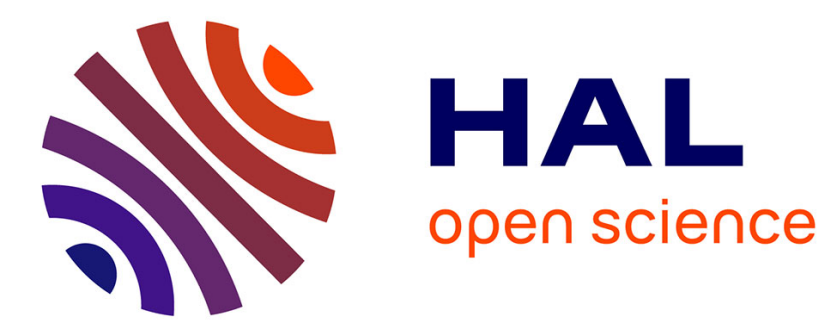

\title{
Un crime sans déviance: le vol en interne comme activité routinière
}

François Bonnet

\section{To cite this version:}

François Bonnet. Un crime sans déviance: le vol en interne comme activité routinière. Revue française de sociologie, 2008, 49 (2), pp.331-350. halshs-00879308

\section{HAL Id: halshs-00879308 \\ https://shs.hal.science/halshs-00879308}

Submitted on 4 Nov 2013

HAL is a multi-disciplinary open access archive for the deposit and dissemination of scientific research documents, whether they are published or not. The documents may come from teaching and research institutions in France or abroad, or from public or private research centers.
L'archive ouverte pluridisciplinaire HAL, est destinée au dépôt et à la diffusion de documents scientifiques de niveau recherche, publiés ou non, émanant des établissements d'enseignement et de recherche français ou étrangers, des laboratoires publics ou privés. 


\section{François Bonnet, «Un crime sans déviance : le vol en interne comme activité routinière », Revue française de sociologie, vol. 49, $\mathrm{n}^{\circ}$, avril-juin 2008, pp. 331-350}

OFCE, Sciences Po

frabonnet@gmail.com

Center for Urban Research and Policy, Columbia

University

1

Résumé : Le vol en interne - les vols commis par les employés sur leur lieu de travail - représente une masse considérable de délits. La littérature économique et de management n'analyse le vol en interne que pour se demander comment le réduire; la littérature sociologique en fait un acte de résistance contre l'injustice au travail. Dans ce travail, on replace le vol en interne dans le cadre de la routine activity approach (un courant 'choix rationnel' de la criminologie américaine) à partir d'une enquête dans les secteurs de la grande distribution et du nettoyage. Analyser le vol en interne comme une délinquance routinière permet de voir que celui-ci est moins une déviance par rapport à une norme qu'une fonction des opportunités et de la surveillance. La théorie du principal-agent et des coûts d'agence est mobilisée pour comprendre que la surveillance du vol s'inscrit moins dans une logique de prévention/répression que de tolérance/acceptation de ce qu'on ne peut pas surveiller. Délinquance routinière, le vol en interne provoque moins le scandale qu'il n'est susceptible d'être instrumentalisé par ses victimes les employeurs pouvant utiliser le vol comme prétexte pour le licenciement. Les enjeux théoriques de la routine activity approach sont discutés en conclusion.

\section{Introduction : définition et ampleur du vol en interne}

Dans les bureaux, les salariés passent des coups de téléphones personnels, font des photocopies et ramènent des fournitures pour leurs enfants ; dans les magasins, ils volent dans les rayons et dans la caisse ; dans les usines, ils dérobent des outils et du matériel. Ceux qui les surveillent, les agents de sécurité, volent aussi, car il n'y a personne pour les surveiller. Au bout de la chaîne, les patrons volent encore, par exemple en commettant des abus de biens sociaux; ils sont souvent les mieux placés pour voler leur propre entreprise. La criminalité des patrons est bien connue en sociologie (Sutherland 1949, Shover et Wright 2000), peut-être parce qu'elle suscite plus de scandale que celle des salariés. Dans cet article, on analysera les vols commis par les salariés comme une activité routinière inscrite dans des relations asymétriques, avec l'idée qu'analytiquement, le vol des employés, le vol des agents de sécurité et le vol du patron ne sont pas très différents : tous volent parce qu'ils le peuvent.

\footnotetext{
${ }^{1}$ Mes remerciements au comité de rédaction de la RFS, à Hugo Bertillot, Patrick Le Galès, Clément Théry et tout particulièrement à Naïma Makri, qui ont subi des versions précédentes de cet article.
} 
Le vol en interne, appelée aussi «fraude interne », désigne les vols réalisés par les salariés sur leur lieu de travail pour leur propre compte. Le «vol» est un terme très général, qui englobe le vol d'argent, de biens, de services (téléphone, Internet) et d'informations (Lipman et McGraw 1988 : 53-54). Cette définition n'englobe pas le vol de temps, comme le fait de prendre des pauses abusives, de bavarder avec ses collègues ou de faire semblant de travailler devant son ordinateur ${ }^{1}$. Le terme « vol en interne » ressort du vocabulaire patronal et n'a de sens que par l'opposition avec les vols «en externe », c'est-à-dire les vols commis par les clients. La criminologie américaine dispose d'une terminologie plus précise. Elle distingue d'abord plusieurs grandes catégories de criminalité : le street crime (les agressions et les vols), le domestic crime (les violences conjugales) et le white-collar crime (les crimes ${ }^{2}$ commis autour du lieu de travail). $\mathrm{Au}$ sein de la catégorie générique white-collar crime, il faut distinguer le corporate crime - ce que nous appelons la délinquance en col blanc, c'est-à-dire les crimes commis par les patrons et les cadres à l'encontre des salariés ou des clients, de l'occupational crime, qui concerne spécifiquement la criminalité des salariés contre l'entreprise. Parmi les délinquances des salariés, la criminologie américaine parle d'employee theft pour désigner le vol en interne, ou d'embezzlement, qui induit l'idée que les salariés abusent de la confiance que l'encadrement place en eux (Beirne et Messerschmidt 2006 : 195 et sq. ; Barkan 2001 : 330 et sq; Siegel $2003: 393$ ).

Comme toute forme de déviance, l'ampleur du vol en interne est difficile à estimer. Les voleurs ne cherchent pas à se faire connaître et les victimes portent rarement plainte. Dans le monde du commerce, les gérants raisonnent en termes de «démarque inconnue ». La démarque inconnue représente l'ensemble des pertes inexpliquées: les vols commis par les clients, les vols commis par les employés, et les erreurs administratives. Selon la sagesse patronale, «la démarque c'est un tiers / un tiers / un tiers »: il y a autant de vol en interne que de vol commis par la clientèle (Shapland, 1995 : 323). Ocqueteau et Pottier (1995 : 120123) estiment à seulement $20 \%$ la part des vols «en externe » dans la démarque inconnue dans le supermarché qu'ils ont enquêté. Des sociologues et des psychologues ont mené des enquêtes par auto-révélation sur le vol en interne ${ }^{3}$. Ces enquêtes montrent de manière assez stable qu'environ un tiers des salariés admettent voler plus ou moins régulièrement leur entreprise (Hollinger et Clark 1983 : 401, Bozon et Lemel 1989 : 116, Kwok et al. 2005 : 463 ; voir Bonnet 2007 pour une présentation plus complète de ces enquêtes). Les estimations du coût du vol en interne sont très variables. Des économistes estiment que les transferts de richesse de l'entreprise vers le salarié indélicat seraient chaque année

\footnotetext{
${ }^{1}$ Le vol de temps est considéré par les entreprises comme un grave problème ; une part importante de la productivité des enseignes Wal-Mart aux Etats-Unis proviendrait de la capacité de l'encadrement à empêcher les salariés de «gaspiller » du temps de travail (Ehrenreich, 2002).

${ }^{2}$ Le droit français distingue les crimes, les délits et les contraventions en fonction de la gravité de l'infraction. La tradition sociologique depuis Durkheim considère cette distinction comme purement arbitraire et administrative. Son seul intérêt est de délimiter les compétences des différentes juridictions (assises, tribunal correctionnel ou tribunal de police). La sociologie utilise le mot « crime » pour désigner indifféremment toutes les infractions.

${ }^{3}$ Dans les enquêtes par auto-révélation, on demande aux enquêtés eux-mêmes de reporter leurs crimes et délits (au contraire des enquêtes de victimation, où on demande aux enquêtés de reporter les crimes et délits subis).
} 
aux Etats-Unis compris entre 15 et 56 milliards de dollars, contre 4 milliards de dollars pour la criminalité ordinaire (street crime) (Dickens et al., 1989).

La plupart des travaux sur le vol en interne portent sur la recherche des causes de cette délinquance. On étudiera ici un autre aspect de la question. L'enjeu de cet article est d'analyser le vol en interne comme une activité routinière, mieux comprise en recourant à la théorie du principal-agent qu'à celle des causes sociales de la délinquance (1): le vol en interne est tellement répandu et si intégré aux relations de travail qu'il constitue un cas parfait de "déviance » qui n'en est pas une et de "crime » qui ne provoque pas le scandale. Après avoir présenté le cadre méthodologique (2) - une enquête qualitative centrée sur les mondes de la grande distribution et du nettoyage -, on dégagera les propriétés empiriques d'une conceptualisation en termes d'activité routinière. Ces propriétés sont au nombre de trois : (3) le vol en interne est essentiellement fonction des opportunités qui se présentent au salarié, et de sa capacité à commettre son forfait en déjouant la surveillance ; (4) ladite surveillance du vol en interne consiste souvent à tolérer des larcins mineurs, à décriminaliser les vols inévitables ; (5) le vol en interne est tellement intégré aux relations professionnelles qu'il est instrumentalisé par les patrons contre les salariés, essentiellement comme prétexte pour des licenciements. En conclusion, on reviendra sur les enjeux théoriques du cadre d'analyse de l'activité routinière.

\section{Le problème sociologique : au-delà de l'étiologie}

Ce travail ne porte pas sur l'étiologie du vol en interne. Le vol en interne a été expliqué par les professionnels de la sécurité et du management comme découlant de l'avidité naturelle des êtres humains (Astor, 1972; Lipman et McGraw, 1988 ; Dickens et al., 1989 ; Kwok et al., 2005) ou de leur faiblesse morale (Merriam, 1977 ; Terris, 1979 ; Bernardin et Cooke, 1993 ; Gibson et Wright, 2001 ; Blount, 2003). Il a été analysé par les sociologues comme un moyen de résistance contre l'injustice de la condition salariale et comme moyen de rétorsion à l'oppression capitaliste (Baumgartner, 1984 ; Tucker, 1989 ; 1993 ; 1999 ; Ackroyd et Thompson, 1999 : 31-52 ; Greenberg, 1990 ; Morrill et al., 2003 : 394-397 ; Hodson, 2004 ; Roscigno et Hodson, 2004: 16) ${ }^{1}$. En fait, déterminer la cause de chaque larcin spécifique est une question empirique; et le chercheur peut décider ou non de prendre au sérieux les justifications de leurs sujets. Par exemple, des chercheurs en management confrontés à des salariés qui disent voler par sentiment d'injustice ont analysé ces discours comme des rationalisations, des simples prétextes (Bernardin et Cooke, 1993 : 1098 ; Kwok et $a l ., 2005: 457$ ), ce qui rend les questionnements sur la signification subjective du vols pour les voleurs comme pour les volés problématique. Les causes du crime sont multiples, complexes et dépendent en fin de compte des préférences idéologiques de celui qui les étudie. Pour la même raison, il est moins intéressant de partir du principe que le vol en interne fait l'objet d'une criminalisation abusive de la part de l'encadrement, que de chercher à comprendre dans quel contexte certains vols sont criminalisés et dans quels autres ils ont tolérés.

L'intérêt du vol en interne pour la sociologie est qu'il s'agit d'une délinquance routinière. Le concept de délinquance routinière provient d'un

\footnotetext{
${ }^{1}$ Pour une analyse plus détaillée de la littérature sur le vol en interne, voir Bonnet (2007).
} 
courant de la criminologie américaine 'choix rationnel' appelé routine activity approach ou criminology of everyday life. Cette perspective a été développée par Cohen et Felson (1979) pour expliquer le paradoxe majeur de la sociologie de la déviance de la fin des années 1970 : la concomitance de la société d'abondance et de la forte augmentation de la criminalité. Aux Etats-Unis, au cours des deux décennies 1960-1970, le PIB croissait de plus de 4\% par an, tandis que Lyndon Johnson mettait en œuvre la Great Society avec les programmes sociaux Medicare et Medicaid. Au cours de la même période, les vols augmentaient de $263 \%$, les cambriolages de $200 \%$, les agressions de $164 \%$, les viols de $174 \%$, et les meurtres de $188 \%$ (Cohen et Felson, 1979). Pendant les vingt-cinq années de forte croissance économique et de forte réduction de la pauvreté et des inégalités, les sociétés occidentales ont vu les vols puis les violences augmenter. Entre 1945 et 1984 en France, le nombre de vols pour 1000 habitants est passé de 4,5 à 43 (Lagrange, 2003 : 15). Entre 1950 et 1988, en Suède, un pays avec un Étatprovidence efficace, le nombre de crimes violents est multiplié par 5, le nombre de cambriolages par 7, et le nombre de vols par 20 (Felson, 1994 : 12).

Jusqu'alors, les théories de la déviance étaient dominées par le paradigme des causes sociales : «un phénomène 'pathologique' comme le crime doit être expliqué par une condition pathologique comme l'aliénation, la pauvreté, les dérèglements familiaux ou l'oppression » (Rock, 2002: 60-61). Pour Cohen et Felson (1979 : 591), l'augmentation de la criminalité après la Deuxième Guerre mondiale s'explique par deux facteurs. D'une part la quantité de biens en circulation augmente de façon si considérable que les opportunités de prédation se multiplient en conséquence ; d'autre part la spécialisation fonctionnelle des espaces urbains et les rythmes synchronisés de travail facilitent grandement le travail des voleurs. En somme, «[p]lutôt que de considérer le predatory crime comme une simple indicateur de disruption sociale, on pourrait le considérer comme un produit dérivé de la liberté et de la prospérité dans la mesure où il se manifeste dans les activités routinières de la vie quotidienne » (Cohen et Felson, 1979 : 605). Le crime est inscrit dans la normalité des sociétés contemporaines et personne n'est prêt à revenir sur ses causes profondes, c'est-à-dire la prospérité et la liberté de mouvement.

Pour la routine activity approach, l'étude du crime doit se focaliser sur le crime tel qu'il est en train de se commettre et non sur le contexte structurel. Pour qu'un crime soit commis, il suffit qu'une opportunité sans risques se présente à un individu. Cet individu n'est pas nécessairement machiavélique, désocialisé ou pauvre, et l'acte délinquant dépend avant tout des circonstances pratiques. Le crime peut alors être conçu comme une activité dépendante de choix rationnels qui s'inscrit dans la routine de la société de consommation. Cette approche résolument rational choice diffère cependant de l'économie du crime (pour un aperçu, Ehrlich 1996). Pour Cohen et Felson, la routine activity approach est une critique de l'approche de Gary Becker (1968) : il s'agit de sociologiser un cadre d'analyse néoclassique perçu comme trop abstrait. Dans ce travail, je me propose de montrer la portée heuristique d'une approche en termes de choix rationnels sur un sujet traditionnellement analysé du point de vue des normes sociales ou du constructivisme. Le propos n'est évidemment pas de soutenir que tout vol en interne doit être analysé comme dépendant uniquement de choix rationnels, et encore moins de faire comme si tous les éléments empiriques mobilisés dans ce 
travail devaient valider une théorie ; il s'agit plutôt de montrer la pertinence de cette approche et sa capacité à illuminer un phénomène obscur. L'un des enjeux de ce choix théorique est de montrer que la théorie du choix rationnel appliquée au crime ne se résume pas, en sociologie criminelle, à légitimer les approches conservatrices du contrôle de la délinquance. Au contraire, une perspective strictement individualiste et mobilisant des concepts issus de la microéconomie comme celui du principal-agent permet de voir comment le vol en interne est instrumentalisé par l'encadrement contre les salariés.

L'idée que le crime soit normal, routinier et intégré aux relations sociales quotidiennes est fructueuse pour la sociologie du vol en interne. Etudier le vol en interne comme une activité routinière, c'est rompre avec l'idée que le vol est forcément fonction de la malignité morale, de la pauvreté ou de la volonté de résistance. Montrer que la surveillance du vol en interne se résume souvent à accorder des avantages, c'est tenir compte du fait que cette surveillance consiste moins à prévenir et/ou réprimer qu'à tolérer un niveau acceptable de déviance pour réduire le risque que des vols plus graves soient; et comprendre comment le vol en interne peut être instrumentalisé, c'est accepter d'envisager la déviance autrement que comme une nuisance par essence sous-optimale qui doit provoquer un rejet viscéral chez les individus bien socialisés. Mais avant cela, on va présenter les données sur lesquelles ce travail s'appuie.

\section{Cadrage méthodologique : une enquête qualitative}

Ce travail s'appuie au départ sur les données produites dans le cadre d'une recherche sur le contrôle de la délinquance dans les centres commerciaux en France et en Italie. 45 entretiens ont été réalisés auprès de policiers, agents de sécurité, directeurs de magasins, employés, syndicalistes, travailleurs sociaux dans deux centres commerciaux de deux grandes villes, une française et une italienne. Ce travail n'est pas une comparaison: il ne s'agit pas de donner au contexte national une quelconque portée explicative. Le vol en interne tel que traité dans ce travail n'était pas le sujet principal des entretiens, mais l'un des thèmes parmi d'autres sur la question générale du contrôle de la délinquance.

\begin{tabular}{|l|l|l|}
\hline Terrain & $\begin{array}{l}\text { Nombre } \\
\text { d'entretiens }\end{array}$ & Personnes interviewées \\
\hline $\begin{array}{l}\text { Centre } \\
\text { commercial } \\
\text { lyonnais }\end{array}$ & $\begin{array}{l}\text { entretiens, } \\
32 \\
\text { personnes } \\
\text { interviewées }\end{array}$ & $\begin{array}{l}\text { Direction du centre : le responsable de la sécurité et le } \\
\text { médiateur } \\
\text { Commerçants : six directeurs, sous-directeurs ou gérants ; } \\
\text { trois responsables de la sécurité ou chefs d'équipe de } \\
\text { sécurité } \\
\text { Employés : sept employés } \\
\text { Policiers : le chef de poste et six policiers (dont deux } \\
\text { entretiens avec deux fonctionnaires) } \\
\text { Syndicalistes : cinq syndicalistes du commerce (dont un } \\
\text { entretien avec deux syndicalistes) et deux de la sécurité. }\end{array}$ \\
\hline $\begin{array}{l}\text { Centre } \\
\text { commercial } \\
\text { milanais }\end{array}$ & $\begin{array}{l}16 \\
\text { entretiens, } \\
13\end{array}$ & $\begin{array}{l}\text { Direction du centre : la directrice du centre commercial } \\
\text { Agents de sécurité : le chef d'équipe (deux fois) et un agent } \\
\text { pe sécurité } \\
\text { interviewées } \\
\text { Vendeurs : deux caissières (syndiquées) de l'hypermarché et } \\
\text { une employée d'un petit commerce } \\
\text { Syndicaliste : deux syndicalistes de la sécurité privée, un } \\
\text { syndicaliste du nettoyage, trois syndicalistes du commerce }\end{array}$ \\
\hline
\end{tabular}




\begin{tabular}{|l|l|l|}
\hline & & (dont un trois fois) \\
\hline
\end{tabular}

Toutes ces données concernent principalement trois milieux professionnels : la grande distribution, le secteur du nettoyage et dans une moindre mesure celui des salles de cinéma. Il s'agit de secteurs féminins, peu syndiqués, mal payés, avec des perspectives limitées dans l'évolution de carrière. Des investigations complémentaires dans d'autres secteurs sont nécessaires pour savoir si et en quoi le vol en interne et sa gestion par l'encadrement diffèrent selon le milieu professionnel.

Pour que des salariés détaillent leurs pratiques délictueuses, il faut un rapport de confiance entre enquêteur et enquêté. Les salariés que j'ai rencontrés par l'intermédiaire de l'encadrement se sont montrés méfiants et cela m'a interdit d'aborder le sujet de façon satisfaisante avec eux. Le personnel de sécurité encadrement, chef d'équipes, agents de sécurité, fonctionnaires de police - a été plus pertinent, notamment sur la surveillance du vol en interne. Instaurer la confiance était dans ce cas de figure moins décisif qu'avec les salariés. Selon toute vraisemblance, les agents de sécurité volent aussi ; les syndicalistes italiens les en accusent d'ailleurs avec véhémence. Mais il était irréaliste d'espérer que les agents de sécurité me parlent de leurs pratiques délinquantes. Au cours de l'enquête, il apparaissait que les interviewés les plus prolixes sur le vol en interne étaient ceux qui avaient été contactés par l'intermédiaire de proches ${ }^{1}$.

Dans un deuxième temps, j'ai donc poursuivi les investigations directement auprès de proches qui avaient des expériences du monde du travail dans les secteurs de la grande distribution, des salles de cinéma et du nettoyage. 3 interviewés-clé m'ont expliqué en détail leurs pratiques au cours de nombreuses conversations, et m'ont introduit auprès de 9 autres interviewés. Le principe de ces entretiens - en fait, des conversations informelles sur plusieurs semaines était de profiter de la confiance établie par les liens personnels pour recueillir le maximum d'informations concrètes sur les vols. Pour ces 12 interactions d'enquête, le vol en interne était le sujet unique de préoccupation. Dans les développements qui suivent, je ferai référence à ces sources par la mention [entretien]. La mention [entretien] permet d'appuyer mon propos sur un travail empirique sans reconstruire ex post et de façon artificielle les discussions informelles que j'ai eues avec ces acteurs.

\begin{tabular}{|l|c|l|}
\hline Secteur & $\begin{array}{l}\text { Nombre } \\
\text { d'informateurs }\end{array}$ & Qualité \\
\hline Distribution & 5 & $\begin{array}{l}\text { Trois caissières dans des grandes surfaces } \\
\text { Deux vendeuses en boutique }\end{array}$ \\
\hline Nettoyage & 4 & Quatre femmes de ménage dans le nettoyage industriel \\
\hline $\begin{array}{l}\text { Salle de } \\
\text { cinéma }\end{array}$ & 3 & Trois vendeuses / ouvreuses \\
\hline
\end{tabular}

\footnotetext{
${ }^{1}$ La question du recours aux proches pour produire des données sociologiques est controversée (voir par exemple Mayer 1995 : 360). Une méthodologie «pure » impliquerait de s'en passer, puisque les proches peuvent introduire toutes sortes de biais.
} 
Concernant le statut des données empiriques dans ce travail, il importe de comprendre que je ne cherche pas à prouver empiriquement que le vol en interne est une activité routinière. Les données statistiques et l'expérience commune suffisent à montrer que le vol en interne est largement répandu et intégré au fonctionnement régulier du monde du travail. Il s'agit de tirer les conséquences empiriques et théoriques d'une analyse du vol en interne comme activité routinière, en partant de quelques cas empiriques spécifiques dont la portée est nécessairement limitée. Les données empiriques mobilisées ne revendiquent pas une prétention à la validité systématique : elles servent à étayer empiriquement des mécanismes liés à la qualité du vol en interne de 'déviance' qui n'en est pas une et de 'crime' qui ne provoque pas le scandale.

\section{Le vol en interne : une question d'opportunités}

Dans une conception du vol en interne comme activité routinière, l'acte délinquant n'est pas la conséquence d'une cause pathologique, mais dépend de la capacité du salarié à profiter de sa «connaissance intime du système de surveillance » (Joseph cité in Anteby 2003 : 467) pour exploiter des opportunités qui se présentent à lui.

\section{A chaque type de travail, son répertoire de vols}

Mars (1982) a observé que la façon dont les salariés volent dépend essentiellement du type de leur activité. Il distingue quatre types de métiers auxquels sont associés des formes particulières de vol, selon que le travailleur soit isolé ou en groupe, et qu'il soit autonome ou non.

\begin{tabular}{c|c}
\multicolumn{2}{c}{ Travail isolé } \\
Autonomie - $\begin{array}{c}\text { Surveillance étroite et salariés } \\
\text { isolés sur le lieu de travail : } \\
\text { caissières, ouvriers à la chaîne }\end{array}$ & $\begin{array}{c}\text { Forte autonomie et salariés } \\
\text { travaillant seuls : cadres, } \\
\text { journalistes }\end{array}$ \\
\cline { 2 - 3 } $\begin{array}{c}\text { Surveillance étroite et travail en } \\
\text { groupe : serveurs, personnel } \\
\text { hôtelier }\end{array}$ & $\begin{array}{c}\text { Relative autonomie et travail en } \\
\text { groupe }: \text { dockers, bagagistes }\end{array}$ \\
Travail en groupe
\end{tabular}

Autonomie

Les salariés isolés et peu autonomes dans leur travail, comme les caissières dans les supermarchés ou certains ouvriers, ne peuvent organiser des combines élaborées. A l'inverse, les caissières font passer leurs proches à la caisse sans les faire payer, en faisant semblant de faire bipper le code barre [entretien]. Ces salariés sont par ailleurs les spécialistes de l'absentéisme (Mars 1982 : 6688). Tous les salariés qui contrôlent des accès, comme dans les cinémas ou les établissements de loisir, peuvent s'arranger pour faire passer leurs proches soit gratuitement, soit à un tarif réduit [entretien]. Très surveillés, ces salariés ont peu d'opportunités ; ils doivent donc savoir les exploiter à bon escient, souvent avec la complicité de proches.

Les employés qui travaillent en groupe et sont peu autonomes, comme les serveurs dans les cafés et le personnel hôtelier, comptent sur leurs collègues pour fermer les yeux sur leurs larcins personnels. Les femmes de ménages des 
sociétés de nettoyage dans les bureaux utilisent la faible surveillance dont elles font l'objet (un chef pour une équipe de plus de 10 personnes éparpillées sur plusieurs étages) pour chaparder des fournitures scolaires. Elles utilisent aussi frauduleusement les lignes téléphoniques sur les bureaux. Elles éteignent la lumière de la pièce dans laquelle elles sont, pour ne pas attirer l'attention. Elles prennent soin de composer un numéro au hasard après avoir raccroché ; comme elles appellent souvent à l'étranger, dans leur pays d'origine, elles veulent éviter que les employés des bureaux ne fassent «bis » en croyant composer le dernier numéro qu'ils avaient appelé et tombent malencontreusement sur une famille au Maroc [entretien].

Ceux qui travaillent en groupe et parviennent à développer un peu d'autonomie organisent généralement des combines de groupes. Dans les grandes surfaces de sport (type Décathlon, Go Sport), les magasiniers intérimaires organisent le tri des t-shirts en fonction de leur taille pour enfiler sous leur pull cinq à six t-shirts volés et sortir du magasin tranquillement [entretien]. Dans un dépôt de marchandise en Italie, les salariés s'étaient lancés dans une entreprise organisée de vol et de revente.

(Et le centre de distribution des marchandises ?) C'est le dépôt de toutes les marchandises que vendent les supermarchés. Un groupe de travailleurs a volé énormément de marchandises, ils s'étaient mis ensemble, avaient un accord avec les routiers, et ils ont volé pour des millions d'euros. Des cartes téléphoniques, du safran. Une caisse de safran ça coûte $200000 €$. Quand l'entreprise s'en est rendu compte, sur quatre caisses de safran qui arrivaient dans le centre, trois seulement repartaient dans les magasins. Donc l'entreprise a porté plainte, et la justice a installé secrètement, de nuit, des caméras, sans accord des syndicats et ils ont fait embaucher des faux employés. Ensuite ils ont encerclé le centre avec des fourgons de police, perquisitionné les travailleurs, fouillé leur casier et leurs maisons. Huit travailleurs détenaient des marchandises volées, leurs maisons étaient pleines, et ils revendaient. (Syndicaliste du commerce 1, Italie)

Les salariés qui travaillent seuls et sont indépendants, typiquement les cadres, exploitent leur autonomie aux dépens de l'organisation. Mars prend l'exemple des journalistes qui facturent des fausses notes de frais quand ils vont au restaurant avec leurs amis, ou des cadres qui profitent de leurs voyages professionnels pour faire du tourisme. En outre, ce type de travailleur triche beaucoup sur le nombre d'heures réellement travaillées (Mars 1982 : 40-65).

L'intérêt de la typologie de Mars réside finalement moins dans sa capacité à prédire le type de vol apparenté à un type de travail mais à montrer que tous les types de salariés volent, qu'ils soient seuls ou en groupe, autonomes ou surveillés, bien rémunérés ou mal rémunérés. De fait, les salariés font l'objet d'une surveillance étroite par l'encadrement. 
Analysé comme une délinquance routinière fonction des opportunités, le vol en interne est un cas exemplaire de problème du principal-agent. Le problème du principal-agent a été formalisé en microéconomie (pour une analyse sociologique : Shapiro 2005). Il sert à caractériser les propriétés d'une situation d'information asymétrique entre deux acteurs qui rentrent dans une relation d'échange. L'exemple fictif est celui du principal (d'un collège) qui donne un travail à accomplir à son agent (d'entretien), travail que le principal juge évidemment utile et nécessaire, mais que l'agent peut considérer comme fatiguant et pénible. L'agent est donc porté à ne pas faire son travail aussi bien qu'il le pourrait, en tous cas comme le souhaiterait le principal. Le problème de la surveillance est donc crucial : le principal ne peut surveiller tout ce que fait son agent, parce que cela est trop coûteux ou impossible. Il y a donc un problème de base dans la relation, puisque le principal ne peut jamais être sûr que l'agent fait bien son travail, tout en devant continuer à le payer. Toute relation hiérarchique dans le monde du travail est de nature principal-agent; le vol en en interne s'inscrit pleinement dans cette structure de relation. D'un côté, les salariés élaborent des stratégies inventives pour profiter des opportunités, informées de leur connaissance des systèmes de sécurité, pour voler l'entreprise; de l'autre, l'encadrement surveille pour limiter les pertes. Certaines tactiques de surveillance sont triviales, d'autres moins. Pour limiter le vol en interne, des magasins font fouiller systématiquement tous les salariés quand ils quittent le travail, les obligeant à emprunter une autre sortie que celle de la clientèle.

On respecte toutes les règles internes, les règles de contrôle, les règles de dissuasion. Y'a moins de tentation. Quand on est dans ces structures, on a des outils de contrôle puissants, on peut tout vérifier. Je vois par exemple ce que font les hôtesses. Tous les jours je surveille, si je sens qu'il y a une anomalie je vais être à l'affût. (Directeur, grande surface sport $B$, centre commercial français)

Dans les grands magasins, les agents de sécurité sont présents bien avant que les clients n'arrivent, pour surveiller les employés du nettoyage et les personnes chargées de l'approvisionnement, et vérifier qu'ils ne volent pas dans les rayons.

$Q$ - Les agents de sécurité...

A - Ici il y en a qui commencent de bonne heure, à 7 heures il est là, en même temps il y a les femmes de ménages, pour le service nettoyage.

$Q-$ Alors ils surveillent les femmes de ménage?

$B$-Automatiquement.

A - Et puis il y a les gens qui viennent livrer. (Deux syndicalistes, grande surface luxe, centre commercial français)

Les salariées du nettoyage sont toujours plus suspectes que les autres de voler. Elles exercent un métier peu gratifiant, mal payé, au contact le plus direct des biens de l'employeur, que celui-ci soit un particulier ou une entreprise. La surveillance du vol et la surveillance de la productivité des femmes de ménage vont de pair. Les employeurs peuvent cacher sous un tapis un billet de 20 euros 
pour s'assurer de l'honnêteté de l'employée, qui sera remerciée si elle ne rapporte pas l'argent [entretien]. L'agent de maîtrise qui supervise une équipe de nettoyage industriel peut cacher des petits morceaux de papier derrière la tuyauterie des toilettes, pour vérifier que son employée est allée nettoyer jusque là [entretien]. Les salariées du nettoyage sont les plus souvent accusées de vol quand le coupable est indécidable; elles font des suspects probables, et permettent de rejeter à l'extérieur de l'organisation la possibilité d'une malfaisance interne.

Les employeurs ne se contentent pas de surveiller leurs employés. La surveillance du personnel est parfois renforcée par la séparation stricte du personnel des magasins et des agents de sécurité. Dans le centre commercial français étudié, les agents de sécurité des magasins sont quasiment tous salariés par une entreprise indépendante du magasin qui loue les services de ses employés. Sous-traiter la gestion des agents de sécurité permet d'établir une séparation nette entre le personnel du magasin chargé d'approvisionner les rayons ou de vendre les denrées, et le personnel de sécurité chargé de surveiller clients et personnels.

Les agents de sécurité ils ne doivent pas sympathiser avec le personnel, la démarque c'est un tiers / un tiers / un tiers, on a plein de saisonniers qui ne sont là que pour un mois ou deux. Chaque membre du personnel doit présenter son sac en sortant, c'est purement de la prévention, pour pas que les gens se disent au bout de deux semaines "ah c'est bon je peux prendre un truc”, je veux pas qu'entre le personnel et les agents ce soit copain-copain, qu'ils fassent la fête ensemble, sinon comment ils surveillent après? (Sous-directeur, grande surface sport A, centre commercial français)

Les agents de sécurité ont pour consigne de ne pas parler avec le personnel du magasin. Le respect de cette règle est renforcé d'une part par la mauvaise image que les agents de sécurité portent en tant que profession et d'autre part par l'altérité qui existe souvent entre agents de sécurité et personnel. Nombre d'agents sont africains et ne maîtrisent pas le français.

Ici la séparation est moins stricte entre le personnel *** et le personnel de sécurité, ce qui est une bonne chose. Mais faut pas fricoter, faut pas faire ami-ami, parce qu'on les surveille aussi, sur le vol et sur le respect des procédures. (Responsable de la sécurité, grande surface loisir, centre commercial français)

Une autre tactique est l'embauche de «prévols », des agents de sécurité en civil qui se font passer pour des clients et surveillent discrètement les agissements des clients comme des employés. L'efficacité du recours aux prévols s'est accrue avec l'accélération du turn-over dans la grande distribution: les salariés qui restent moins longtemps en poste sont moins capables de repérer les faux clients et sont donc moins à même d'informer leurs collègues. Evidemment, toutes ces solutions de surveillance ont un coût.

L'intérêt d'une conceptualisation en termes de principal-agent est d'abord d'écarter l'idée de déviance du vol en interne : voler son entreprise n'est pas un problème de violation d'une norme, mais la conséquence logique d'une structure de relation qui permet et incite les comportements opportunistes. C'est 
aussi de voir que le vol est d'autant plus un problème pour le principal (l'encadrement) que la surveillance des agents (les salariés) a un coût élevé : le salaire des agents de sécurité et le temps de travail des chefs. Dans la théorie du principal-agent, le coût financier de la surveillance n'est que l'un des multiples coûts d'agence (agency costs), que l'on peut définir comme tous les coûts, problèmes et soucis qui proviennent de l'existence de la structure de relation en principal-agent. Ainsi, la surveillance n'est pas le seul moyen de minimiser l'occurrence du vol en interne : l'encadrement peut aussi tolérer les vols les plus difficilement contrôlables.

\section{Tolérer les petits vols pour éviter de surveiller à perte}

Comment optimiser la surveillance du vol en interne ? Des économistes néoclassiques (Dickens et al. 1989) ont observé que dans le monde réel, les entreprises dépensent des sommes considérables pour la surveillance. Pourtant, les solutions pareto-optimales au problème du vol en interne seraient soit (a) de payer des salaires d'efficience aux salariés, qui seraient incités à ne pas risquer de perdre leur travail, soit (b) de surveiller au minimum mais d'infliger des peines d'une sévérité extrême, afin de dissuader à faible coût. D'une part, le problème des salaires d'efficience découle du principe même de la structure de relation principal-agent: l'employeur ne peut se permettre de payer des salaires d'efficience à tous les salariés sans savoir qui est vertueux et qui ne l'est pas, sous peine d'inciter tout le monde à voler - cas classique de moral hazard. D'autre part, le problème de la sévérité excessive est qu'elle peut être limitée par le droit du travail ; qu'elle peut heurter le sentiment tacite de justice des salariés, et donc leur motivation; surtout, la possibilité d' «erreurs judiciaires» de la part de l'entreprise risque d'inciter les salariés à passer plus de temps à se prémunir contre une possible erreur qu'à travailler pour l'entreprise. En d'autres termes, la surveillance du vol en interne induit des coûts d'agence qui dépassent le strict coût financier des moyens technologiques et humains de cette surveillance.

Du coup, une tactique courante est non pas de surveiller le vol en interne pour punir les salariés-voleurs, mais de normaliser l'inconduite, de la décriminaliser, de façon à éviter les coûts liés à la surveillance et aux conflits. Cette normalisation se traduit le plus souvent sous la forme des «petits avantages » en nature accordés aux salariés (Ditton, 1977a et 1977b). Ditton explique que le vol en interne est un triptyque. Le chapardage (pilferage) recouvre toutes les formes traditionnelles de vol; Les combines (fiddle) sont les petites escroqueries quotidiennes que les salariés pratiquent aux dépens des clients (typiquement, mal rendre la monnaie et garder la différence); les «à-côtés » (perks) sont les avantages en nature liés à chaque métier. Pour Ditton, les «àcôtés » sont le plus souvent des tolérances de l'encadrement quand le coût de la surveillance excède celui du dommage.

Quand le vol en interne de chaque employé particulier porte sur des quantités dérisoires et inoffensives, il est irrationnel de tenter de contrôler ce type de comportement. Un exemple de la forme la moins dommageable pour l'entreprise, et la moins contrôlable de vol en interne, est l'autoconsommation de denrées alimentaires par les vendeurs dans les kiosques à sandwiches et à viennoiseries. Les boissons et les marchandises prêtes à la consommation et vendues au détail sont particulièrement attractives pour les employés. 
(Qu'est-ce qui te plaisait dans le travail?) (...) Je

bouffais à l'œil, c'était vachement bon, et comme je suis gourmande... (Vendeuse 2, centre commercial français)

Dans le centre commercial français étudié, il est si difficile et si coûteux pour l'encadrement de se prémunir contre la disparition intempestive de soda et de pâtisseries que le fait de se servir gratuitement dans les denrées destinées à la vente est toléré. L'auto-consommation est alors un «à-côté» du métier de vendeur.

Bah, on est nourri sur place, c'est dans le contrat, y'en a qui prennent des tonnes de trucs à midi pour les remmener le soir chez eux. Des fois, on passe en civil à midi, "tu peux me filer une canette discrétos?", ça reste gentillet, ou le soir on peut emporter deux trois trucs qui restent, mais c'est permis. (Vendeuse 1, centre commercial français)

Le vol en interne, surtout dans sa dimension de chapardage, est imputé en priorité aux travailleurs les moins qualifiés et les moins bien rétribués, pour lesquels le moindre revenu complémentaire peut faire la différence - par exemple, les salariés du nettoyage. Dans les immeubles de bureaux (ou dans les universités), les femmes de ménage sont chargées du remplacement de rouleaux de papier-toilette. Pour éviter que les usagers se retrouvent à court de papier au moment fatidique, les femmes de ménages doivent remplacer les rouleaux avant leur épuisement complet. Bien sûr, elles ont le droit de conserver les chutes des rouleaux de papier-toilette. Comme il serait absurde de déterminer un seuil objectif à partir duquel un rouleau doit être remplacé, les femmes de ménages s'adjugent des chutes généreuses - si bien qu'elles n'achètent jamais de papiertoilette pour leur propre foyer [entretien]. Externalité positive : il ne manque jamais de papier-toilette pour les usagers. Dans ce cas, le caractère minime des pertes ne justifie pas une surveillance coûteuse. Le chapardage de chutes de papier-toilette est un vol mineur légitimé par une tolérance plus ou moins contrainte de l'encadrement. La plupart des à-côtés que les métiers offrent ne sont que des chapardages incontrôlables, que l'encadrement préfère tolérer puisqu'il ne peut les empêcher.

Il en est de même pour les employées d'une papeterie et des caissières des salles de cinéma. Dans une papeterie étudiée, les biens sont en libre service, empilés pour être touchés et manipulés par le public, ce qui maximise les ventes. Les clients volent donc beaucoup, ce qui induit une grande incertitude pour l'employeur: il est impossible d'accuser ses employés avec certitude. Les employées sont des étudiantes en BTS qui invitent leurs amies dans le magasin pour discuter, faire passer le temps et leur montrer les produits qui pourraient les intéresser. Quand les employées doivent faire un cadeau, elles s'assurent d'abord que le produit plait avant de le voler puis de l'offrir. Pour limiter le vol par les employées, le patron a proposé de leur vendre les produits avec un rabais de $40 \%$. Il vend toujours au dessus de sa marge (qui est de plus de $100 \%$ du prix fournisseur) et modifie la structure du calcul risque/avantages de ses employées : elles font une affaire par rapport au prix du marché et ne prennent pas le risque d'être découvertes; si elles volent, elles n'ont aucune excuse [entretiens]. Les exploitants de salle de cinéma distribuent de la même façon des places gratuites aux caissières : plutôt que celles-ci utilisent leur temps de travail pour élaborer des 
dispositifs visant à voler le cinéma pour inviter leurs amis, les exploitants préfèrent abandonner un nombre calculé de places de cinéma [entretiens]. Ils y gagnent en légitimité, peuvent jouer dessus dans la négociation des salaires, et s'assurent que le temps de travail ne sera pas perdu en complots contre l'organisation.

Le vol en interne est souvent toléré lorsqu'il est circonscrit dans les limites du raisonnable, c'est-à-dire inoffensif pour la rentabilité du magasin. Il devient une menace quand il ne se limite plus à de simples chapardages, mais prend les proportions d'une entreprise de prédation. Dans certains magasins, le vol en interne fait partie des rétributions occultes qui attirent jusqu'aux futurs employés. Dans les grandes surfaces de sport (type Décathlon ou Go Sport), qui emploient un important volet d'étudiants en CDD, les anciens salariés qui recommandent le travail à leurs amis mentionnent le fait qu'il est toujours possible de voler des t-shirts ou des balles de tennis [entretien]. Dans un commerce alimentaire d'une gare ferroviaire, la facilité avec laquelle il était possible de voler avait fait naître des vocations :

C'est comme les sandwiches-clubs et les canettes, elles sont en libre service, comme dans les grandes surfaces, on en vend plus, mais en même temps ça multiplie les risques de gratte [vol en interne]. Comme ça il y a des bruits qui courent, il y a des gens qui venaient travailler uniquement pour la gratte! (Syndicaliste du commerce, France)

La tolérance de certains vols et de leur transformation en avantages, en àcôtés montre que la surveillance et le contrôle de la délinquance ne se résument pas à l'alternative prévention / répression. On peut analyser la tolérance des petits vols par l'encadrement comme un arbitrage entre différents coûts d'agence : dans les configurations où les coûts financiers et sociaux de la surveillance sont excessifs, les coûts associés à la tolérance des vols peuvent être plus supportables. Faut-il pour autant associer tous les petits avantages, tous les actes ordinaires d'appropriation au vol en interne ? La réponse est double : d'une part, du point de vue strictement conceptuel, tous ces actes véniels qui font le quotidien de tous les salariés sont des mini-vols contre lesquels l'encadrement ne peut rien et qui sont donc autorisés de fait, mais qui en toute rigueur portent un tort à l'entreprise. D'autre part, il est symétriquement absurde, du point de vue conceptuel, de considérer qu'un acte puisse avoir la moindre dimension délictueuse si aucun système de sanction n'est capable de l'appréhender, ou si la sanction aurait des effets bien pires que personne ne souhaite. En conséquence, la vraie question est plutôt celle du tracé de la frontière entre ce qui fait partie de la régulation normale de l'organisation du travail (ce qui est toléré) et ce qui est considéré comme abusif (ce qui est prohibé). Moins le tracé est explicite, plus les salariés ont de latitude pour jouer sur les ambiguïtés avec mauvaise foi. Le cas du vol en interne montre que la gestion de la criminalité consiste aussi à retracer la frontière entre ce qui est acceptable et ce qui ne l'est pas, et pas seulement à surveiller et punir. En déplaçant cette frontière, en acceptant que les salariés volent un peu (en leur accordant des réductions), les employeurs font le calcul que cela leur coûtera moins que de les surveiller et d'entrer en conflit pour des broutilles qui seront volées de toutes façons. On perd moins à tolérer l'inévitable qu'à apparaître inutilement tatillon et, en fin de compte, faible. 


\section{L'instrumentalisation du vol en interne}

Analyser le vol en interne comme activité routinière et non comme déviance par rapport à une norme suppose de voir en quoi cette activité peut profiter à ses supposées victimes - les employeurs. Mars (1982) et Ditton (1977a) ont observé, sur leurs sites d'enquête respectifs, que l'encadrement tend à fermer les yeux si le dommage n'incombe qu'aux clients. Dans la boulangerie de Ditton, les caissiers ont développé une grande habileté pour ne pas rendre toute la monnaie aux clients et garder la différence. Les arnaques élaborées par les serveurs de café de Mars sont tolérées tant qu'elles ne sont pas grossières. Tolérer les petites combines des employés aux dépens des clients présente un faible coût pour les employeurs : comme il ne s'agit pas d'un système organisé, la faute est individuelle et si un client se plaint, l'employeur aura beau jeu de s'excuser et de punir l'employé. Mais surtout, le vol des clients par les employés permet de comprimer les salaires : les employés qui se constituent un revenu d'appoint de cette manière sont moins enclins à revendiquer une augmentation. Ils ne veulent pas attirer l'attention et préfèrent garder leur marge de manœuvre de négociation sur d'autres aspects des conditions de travail sur lesquelles ils ne peuvent pas tricher, comme l'organisation du planning. Pour Ditton, le vrai scandale politique du vol en interne réside dans la situation de double bind des employés : obligés de voler pour se constituer un revenu décent, mais menacés de licenciement s'ils se font prendre.

Le vol en interne permet en outre de fournir à l'employeur un prétexte pour licencier à moindre frais. Le droit du travail peut obliger le patron à verser de substantielles indemnités au salarié en cas de licenciement pour motif autre que faute grave ${ }^{1}$. Sachant que de nombreux employés volent, les patrons qui veulent licencier des salariés à moindre coût n'ont qu'à attendre que ceux-ci commettent une infraction pour lancer la procédure.

Mais par contre, il y a eu cinq salariés qui se sont fait prendre par la police parce que le patron avait demandé à ce qu'elle installe des caméras, elle a surveillé pendant six mois et puis ils ont interpellé cinq salariés. On n'a pas trouvé ça normal qu'il soit passé par les flics, qu'il laisse faire ça en connaissance de cause, il aurait pu juste organiser un flagrant délit. (...) Enfin on a réussi à éviter le clash entre ceux qui sont contre la gratte et ceux qui grattent, ensuite ça devient un prétexte pour sauter les salariés. (Syndicaliste du commerce, France)

Généralement, la menace de poursuites pénales suffit pour que le salarié présente sa démission sur le champ, ce qui évite à l'entreprise les frais de procédure. Dans le monde du commerce, l'instrumentalisation de la pénalisation du vol en interne permet d'offrir une marge de flexibilité supplémentaire aux entreprises. Cela facilite la mise à la porte des éléments jugés peu productifs ou sur le déclin. Occasionnellement, certaines entreprises ne s'interdisent pas de tendre des pièges.

\footnotetext{
${ }^{1}$ En Italie, le droit du travail oblige les entreprises de plus de 15 salariés à passer devant le juge pour vérification de la validité de chaque licenciement. En France, seuls les salariés qui ont 2 ans et plus d'ancienneté ont droit à des indemnités (2 mois de salaire par 10 années travaillées) ; mais le juge a toute latitude pour augmenter ces indemnités.
} 
Le problème du vol en interne, c'est que les gens se font licencier pour un crayon ou pour un magnétoscope, un vol est un vol mais bon... (...) Il y a aussi des situations d'incitation au vol. Le supermarché fait appel à des sociétés, officiellement de portierati, mais qui en fait envoient des hommes dans les magasins pour tester les caissières. Par exemple tu achètes une bouteille de champagne, 20€, et tu les donnes à la caissière sans attendre qu'elle te donne le ticket de caisse, elle est tentée de prendre les $20 €$ et de pas enregistrer la bouteille de champagne. Ensuite un garde contrôle le faux client, il n'a pas de ticket de caisse, on l'accuse de vol, il accuse la caissière, c'est sa parole contre la sienne, évidemment si c'est une caissière dont on veut se débarrasser on lui propose un accord "à l'amiable", elle démissionne et personne ne découvre que le client est en fait un employé qui la testait. (Syndicaliste du commerce 2, Italie)

Il y a quelques temps, une amie m'a appelé pour me dire qu'elle faisait partie d'une agence qui était employée par le centre commercial, ils font semblant d'être des clients, pour tester les caisses et voir si les caissières volent pas. Mais comme je le savais... (rires) (Caissière 1, centre commercial italien)

Le personnel fait l'objet d'une surveillance de sa productivité autant que de son honnêteté. L'instrumentalisation du vol en interne permet aux entreprises de licencier des salariés indésirables. On mesure alors toute la naïveté de la conception du vol en interne comme acte de résistance contre l'injustice de la condition salariale : assimiler le vol en interne à un acte politique représente le danger de ne pas voir que le vol en interne est instrumentalisé par l'encadrement pour faciliter les licenciements. On comprend aussi pourquoi certains patrons n'ont pas intérêt à problématiser le vol en interne.

Souvent on se sert de cette méthode pour virer les femmes de plus de cinquante ans, qui ont des problèmes, qui prennent des médicaments, des mères seules, qui font souvent des arrêts-maladie. (Syndicaliste du commerce 2, Italie)

L'instrumentalisation du vol en interne par les employeurs a des conséquences théoriques. Durkheim (1988) a affirmé que le crime provoquait un scandale moral tel qu'il permettait de raffermir la solidarité du groupe ; Becker (1985) a défini le crime comme une déviance par rapport à une norme. Le cas du vol en interne analysé comme activité routinière permet de nuancer ces conceptions : les employeurs ne sont pas scandalisés par cette délinquance qu'ils perçoivent moins comme une déviance (un rapport à une norme) qu'une opportunité stratégique de contourner les contraintes d'un système institutionnel à leur profit. On comprend qu'une norme qui peut être enfreinte et instrumentalisée de manière routinière n'en est pas une. On comprend aussi que l'opportunisme et la rouerie ne sont pas l'apanage des agents: les principaux savent être assez

\footnotetext{
${ }^{1}$ Le marché italien de la sécurité privée est dual. Les agents de sécurité classiques sont armés, assermentés et disposent d'un contrat de travail relativement avantageux; en contrepartie, ils doivent être de nationalité italienne et être soumis à de nombreux contrôles. Pour minimiser les coûts, les entreprises emploient de plus en plus des portierati [portiers], c'est-à-dire des agents de sécurité non-assermentés, non-armés, non-contrôlés, souvent immigrés et très mal payés.
} 
malins pour transformer un coût d'agence en solution aux problèmes que leur pose le code du travail.

\section{Conclusion : les enjeux théoriques de la routine activity approach}

Il est rassurant de penser le crime comme une déviance, et ce pour plusieurs raisons. La déviance-comme-écart-à-la-norme nous rappelle la majesté des principes normatifs qui fondent la cohésion de notre groupe d'appartenance. La déviance-comme-exception-au-quotidien nous conforte dans l'illusion d'un monde sûr et bien réglé. La déviance-comme-imposition-arbitraire-d'un-label permet de mettre des concepts sur notre sentiment d'injustice face à l'intolérance des entrepreneurs de morale. En cherchant l'origine de la déviance dans des pathologies sociales, on s'assure de ses causes, de préférence lointaines et utiles à un agenda politique. Et si le crime était une activité banale et routinière ? Le vol en interne se présente comme un défi à la sociologie de la déviance : qu' y a-t-il de déviant à faire ce que tout le monde fait, c'est-à-dire optimiser sa satisfaction par des moyens illégaux ? Comment trouver un lien entre délinquance et pauvreté, délinquance et inégalités, délinquance et couleur de la peau quand, de manière routinière, la plupart des salariés chapardent, dérobent, «empruntent», détournent, quelle que soit leur position dans la hiérarchie, quels que soit leur besoins objectifs, quelle que soit la «normalité » de leur éducation?

Dans cet article, on a montré le bénéfice qu'il y avait à analyser le vol en interne comme une activité routinière. La question des opportunités et de la surveillance montre qu' on ne gagne pas grand chose à penser le vol en interne comme une déviance ; une déviance suppose une norme, et une norme n'en est pas une si sa violation est routinière. Analyser le vol en interne comme problème du principal-agent permet d'évacuer la dimension morale du vol et de penser le vol comme activité routinière. Le fait que les entreprises tendent à décriminaliser pour ne pas être en situation de ne pouvoir ni les surveiller ni les punir en dit long sur le caractère intégré du vol en interne aux relations de travail. La surveillance vise moins à prévenir et à réprimer qu'à retracer la frontière du tolérable et de l'intolérable; la «déviance » n'est pas seulement combattue et réprimée, elle est aussi gérée. Au lieu de provoquer le scandale, le crime peut faire l'objet d'instrumentalisations ingénieuses par ses «victimes» (en fait, des acteurs compétents et réflexifs). Cette analyse d'un crime sans déviance suggère donc de restreindre le domaine de validité du paradigme de la déviance en sociologie criminelle, et d'être plus attentif à une conceptualisation de la délinquance opportuniste comme manifestation de la capacité d'agency des acteurs.

L'analyse en terme d'activité routinière n'est pas sans poser des problèmes théoriques et éthiques. Le principal problème théorique est celui de la dynamique de la définition des crimes. Les théories de l'activité routinière dissolvent la complexité du crime dans une théorie du choix rationnel certes très efficace pour comprendre les mécanismes de surveillance et de contrôle, mais plus maladroite dès que le crime considéré a une dimension morale ou politique. Ces théories tendent à assimiler le crime à une simple nuisance, délégitimant par avance la dimension politique potentielle des conduites délictueuses ; elles figent la définition de ce qui est considéré comme crime. Le problème politique concerne les politiques de sécurité associées à la routine activity approach. En l'espèce, il s'agit de la «prévention situationnelle »(Clarke, 1992 ; 1995). La 
prévention situationnelle recouvre l'ensemble des politiques qui se focalisent sur le calcul effectué par le criminel potentiel au moment de commettre son crime. Les politiques de prévention situationnelle cherchent à élever le coût de participation à l'activité criminelle et à en diminuer les bénéfices pour réduire le crime, en particulier grâce à des solutions techniques, comme les portes blindées, les verrous, la vidéosurveillance, etc.. La prévention situationnelle et ses dérivés «mettent l'accent sur des solutions pragmatiques, fondées sur l'expertise, moralement neutres et rationnellement instrumentales »(Garland, 2001: 182). Elles permettent de mettre en œuvre des «politiques proprettes, pas chères, à petite échelle, faisables et peu sujettes à controverse » (Rock, 2002 : 76). Leur efficacité à court-terme (Pease, 2002 : 956) les rend donc séduisantes mais évacue toute notion de contexte macro.

\section{Bibliographie}

Ackroyd S., Thompson P. (ed.). - 1999, Organizational Misbehavior, Londres : Sage

Anteby M., 2003. - «La "perruque" en usine : approche d'une pratique marginale, illégale et fuyante », Sociologie du Travail, 45 (4), décembre, pp. 453-471

Astor S.D., 1972. - «Who's doing the stealing ?», Management Review, 61, pp. 34-35

Barkan S., 2001. - Criminology. A Sociological Understanding, Second Edition, Upper Saddle River, NJ: Prentice Hall

Baumgartner M. P., 1984. - "Social Control from Below », in Black D., Toward a General Theory of Social Control. Volume 1: Fundamentals, Orlando: Academic Press, «Studies on Law and Social Control », 1984, pp. 303-345

Becker G.S., 1968. - «Crime and Punishment: An Economic Approach », Journal of Political Economy, 76 (2), pp. 169-217

Becker H.S., 1985 (1963) . - Outsiders. Etudes de sociologie de la déviance, Paris : Métailié

Beirne P., Messerschmidt J.W., 2006. - Criminology, Fourth Edition, Los Angeles, CA : Roxbury Publishing Company

Blount E.C., 2003. - Occupational Crime : Deterrence, Investigation, and Reporting in Compliance with Federal Guidelines, Boca Raton : CRC Press

Bonnet F., 2007. - « Le vol en interne : les vols commis par les salariés sur leur lieu de travail », Sociologie du travail, vol. 49, pp. 544-556

Bozon M., Lemel Y., 1989. - «Les petits profits du travail salarié. Moments, produits et plaisirs dérobés », Revue française de sociologie, XXX, pp. 101-127

Cohen L., Felson M., 1979. - «Social Change and Crime Rate Trends: A Routine Activity Approach », American Sociological Review, 44 (4), pp. 588-608

Dickens W.T., Katz L.F., Lang K., Summers L.H., 1989. - « Employee Crime and the Monitoring Puzzle », Journal of Labor Economics, 7 (3), July 1989, pp. 331-347

Ditton J., 1977a. - «Perks, Pilferage, and the Fiddle : The Historical Structure of Invisible Wage », Theory and Society, 4 (1), Spring, pp. 39-71 
Ditton J., 1977b. - Part-Time Crime. An Ethnography of Fiddling and Pilferage, Londres : MacMillan

Durkheim É., 1988 (1895). - Les règles de la méthode sociologique, Paris : Flammarion, «Champs »

Ehrenreich B., 2002, Nickel and Dimed. On (not) Getting by in America, Owl Books

Ehrlich I., 1996. - «Crime, Punishment and the Market for Offenses », Journal of Economic Perpectives, 10 (1), pp. 43-67

Felson M., 1994. - Crime and Everyday Life. Insight and Implications for Society, Thousands Oaks : Pine Forge Press

Felson M., 2002. - Crime and Everyday Life. Third Edition, Thousand Oaks: Sage

Garland D., 2001. - The Culture of Control. Crime and Social Order in Contemporary Society, Chicago : University of Chicago Press, Oxford : Oxford Universty Press

Gibson C., Wright J., 2001. - «Low Self-Control and Coworker Delinquency : A Research Note », Journal of Criminal Justice, 29 (5), pp. 483-501

Gottfredson, M.R., Hirschi T., 1990. - A General Theory of Crime, Stanford : Stanford University Press

Greenberg J., 1990. - «Employee Theft as a Reaction to Underpayment Inequality: The Hidden Costs of Pay Cuts », Journal of Applied Psychology, 75 (5), pp. 561-568

Hodson R., 2004. - «A Meta-Analysis of Workplace Ethnographies. Race, Gender, and Employee Attitudes and Behaviors », Journal of Contemporary Ethnography, 33 (1), February, pp. 4-38

Hollinger R.C., Clark J.P., 1983. - «Deterrence in the Workplace : Perceived Certainty, Perceived Severity, and Employee Theft », Social Forces, 62 (2), December, pp. 398-418

Kwok C.-K., Au W.T., Ho J.M.C., 2005. - « Normative Controls and SelfReported Counterproductive Behaviors in the Workplace in China », Applied Psychology: An International Review, 54 (4), 2005, pp. 456-475

Lagrange H., 2003. - Demandes de sécurité. France, Europe, Etats-Unis, Paris : Le Seuil, «La République des Idées »

Lipman M., McGraw W.R., 1988. - «Employee Theft: A $\$ 40$ Billion Industry », Annals of the American Academy of Political and Social Sciences, vol. 498, July, pp. 51-59

Mangione T.W., Quinn R.P., 1975. - «Job Satisfaction, Counter-Productive Behavior and Drug Use at Work », Journal of Applied Psychology, vol. 60, pp. 114-16

Mars G., 1982. - Cheats at Work. An Anthropology of Workplace Crime, Londres : George Allen and Unwin

Mayer N., 1995. - «L'entretien selon Bourdieu : analyse critique de La misère $d u$ monde », Revue française de sociologie, 36 (2), pp. 355-370

Merriam D., 1977. - «Employee Theft », Criminal Justice Abstracts, 9, pp. 380386

Morrill C., Zald M.N., Rao H., 2003. - « Covert Political Conflict in Organizations : Challenges from Below », Annual Review of Sociology, vol. 29, pp. 391-415 
Ocqueteau F., Pottier M.-L., 1995. - Vigilance et sécurité dans les grandes surfaces, Paris : L'Harmattan

Pease K., 2002. - «Crime Reduction », in Mike Maguire, Rod Morgan, Robert Reiner (dir.), The Oxford Handbook of Criminology, Oxford : Oxford University Press, 2002, $3^{\mathrm{e}}$ edition, pp. 947-979

Rock P., 2002. - «Sociological Theories of Crime», in Mike Maguire, Rod Morgan, Robert Reiner (dir.), The Oxford Handbook of Criminology, Oxford : Oxford University Press, $3^{\mathrm{e}}$ edition, pp. 51-82

Roscigno V.J., Hodson R., 2004. - « The Organizational and Social Foundations of Worker Resistance », American Sociological Review, 69 (1), February, pp. 14-39

Shapland J., 1995. - «Preventing Retail-Sector Crimes », Crime and Justice, vol. 19, pp. 263-342

Shapiro S.P., 2005. - «Agency Theory », Annual Review of Sociology, vol. 31, pp. 263-284

Shover N., J.P. Wright (dir.), 2000. - Crimes of Privilege : Readings in WhiteCollar Crime, Oxford: Oxford University Press

Siegel L.J., 2003. - Criminology, Eighth Edition, Belmont, CA: Wadsworth

Sutherland E.H., 1949. - White Collar Crime, New York : Dryden Press

Terris W., 1979. - «Attitudinal Correlates of Employee Integrity », Journal of Security Administration, vol. 2, pp. 30-39

Tucker J., 1989. - «Employee Theft as Social Control », Deviant Behavior, 10, pp. 319-334

Tucker J., 1993. - «Everyday Forms of Employee Resistance », Sociological Forum, 8 (1), March, pp. 21-45

Tucker J., 1999. - «Worker Deviance as Social Control», Research in the Sociology of Work, Vol. 8, pp. 1-16 